\title{
USO DO CAMPO POTENCIAL NA EXPLORAÇÃO DOS RECURSOS MINERAIS MARINHOS
}

\author{
Marcelo S. Dias ${ }^{1} \&$ Luiz F. S. Braga ${ }^{2}$
}

Received September 21, 2001 / Accepted December 05, 2001

\begin{abstract}
Os métodos potenciais têm sido amplamente utilizados para geofísica de exploração desde o início do último século. Suas aplicações, em geral, foram empregadas no mapeamento regional de grandes estruturas relacionadas a recursos minerais como: falhas de borda da bacia, grabens e horsts, diápiros de sal, profundidade do embasamento e estimativa de fluxo de calor. No entanto, durante a última década, equipamentos de alta resolução (altimetria de satélite, gradiômetros gravimétricos e magnetométricos, magnetômetro de césio, DGPS e computadores potentes) tornaram disponíveis um enorme volume de dados para exploração de recursos minerais marinhos. Atualmente, estes dados têm sido usados para calibração de pequenos contrates de densidade e de susceptibilidade magnética, fundamentais para várias pesquisas em exploração, tais quais: i) correção de velocidade para a sísmica de reflexão 3D e 4D; ii) micro-gravimetria e micro-magnetometria para a geofísica de poço; iii) mapeamento de densidade e porosidade em alta resolução para fluído em poros; iv) mapeamento de estruturas de riscos geotécnicos e v) sulfetos e nódulos polimetálicos em crosta oceânica. Atualmente, uma nova visão dessas aplicações esta sendo utilizada pela indústria de exploração mineral. O objetivo principal deste artigo é o de fazer uma breve revisão sobre o tema e também dar algum suporte teórico para essas aplicações.
\end{abstract}

Palavras-chave: Métodos potenciais; Gravimetria; Magnetometria; Recursos minerais marinhos.

GRAVITY AND MAGNETICS IN MARINE MINERAL EXPLORATION - The potential field methods have been widely used for exploration geophysics since the beginning of the last century. In general, their applications were for preliminary regional mapping of large geological structures related to mineral resources such as: basin boundary faults, grabens and horsts, salt diapirs, basement geometry and heat flow estimates. However, during the last decade, a new branch of equipment (satellite altimeter, gravity and magnetic gradiometers, cesium magnetometers, DGPS and powerful computers) made available an enormous volume of high-resolution data for marine mineral resources exploration. Nowadays, these data are being used for calibrating small density and magnetic susceptibility contrasts that are fundamental for investigating a number of exploration issues: i) velocity correction for $3 D$ and $4 D$ seismic reflection; ii) microgravity and micro-magnetic for core geophysics, iii) high resolution density and porosity for pore fluids; iv) potential hazards structures and v) sulfides and polimetallic nodules in oceanic crust. A new vision of these applications is already in use by the mineral exploration industry. The main goal of this paper is to make a brief review and also give some background on those applications.

Key words: Potential methods; Gravity; Magnetics; Marine mineral resources.

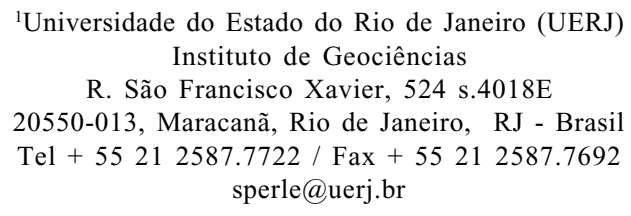

\author{
${ }^{2}$ Observatório Nacional - MCT / Depto. de Geofísica \\ R. Gal. José Cristino, 77 \\ 20921-400, São Cristóvão / Rio de Janeiro, RJ - Brasil \\ braga@dge1.on.br \\ 1.braga@fulgroairborne.com.br
}




\section{INTRODUÇÃO}

Os métodos potenciais foram os primeiros a serem utilizados como ferramenta nos estudos pioneiros de geologia e geofísica, na década de 20, para exploração de recursos minerais (Gibson \& Millegan, 1998). Desde então, a praticidade e o baixo custo dos equipamentos (gravímetros e magnetômetros) permitiu sua ampla utilização para obter anomalias gravimétricas e magnéticas, estimando, respectivamente, a densidade e a susceptibilidade magnética das rochas em subsuperficie. De maneira global, tais métodos permitiram obter as mais importantes evidências sobre a gênese e evolução da litosfera oceânica na década de 50 . Tais evidências foram a base para a formulação da Teoria de Tectônica de Placas, na década de 60, que posteriormente revolucionou as Geociências no século XX.

Na década de 70, após ampla utilização com fins científicos, os métodos potenciais foram os precursores nas pesquisas e exploração de recursos minerais marinhos, principalmente os hidrocarbonetos (petróleo e gás) nas margens continentais (Nettleton, 1976). Foram amplamente utilizados para o mapeamento dos limites das bacias sedimentares e de diversos alvos importantes para a indústria do petróleo (falhas, domos salinos, grabens, horsts, espessura crustal, etc). Nos fins dos 70, Mckenzie (1978) desenvolveu um modelo termo-mecânico e demonstrou, de maneira simples e elegante, como os métodos potenciais poderiam ser utilizados para fins de exploração de petróleo e gás em bacias sedimentares. Este modelo, parametrizado principalmente pela gravimetria, considerando os conceitos de isostasia, revolucionou a indústria do petróleo e foi refinado por diversos pesquisadores sendo amplamente utilizado até os dias de hoje. (Karner \& Watts, 1982; Weissel \& Karner, 1989; Allen \& Allen, 1990; Karner \& Drisscoll, 1993; Sperle, 1997).

Após décadas de exploração do subsolo continental concluiu-se, na década de 80 , que as reservas de recursos minerais nobres tais como: $\mathrm{Ni}$,

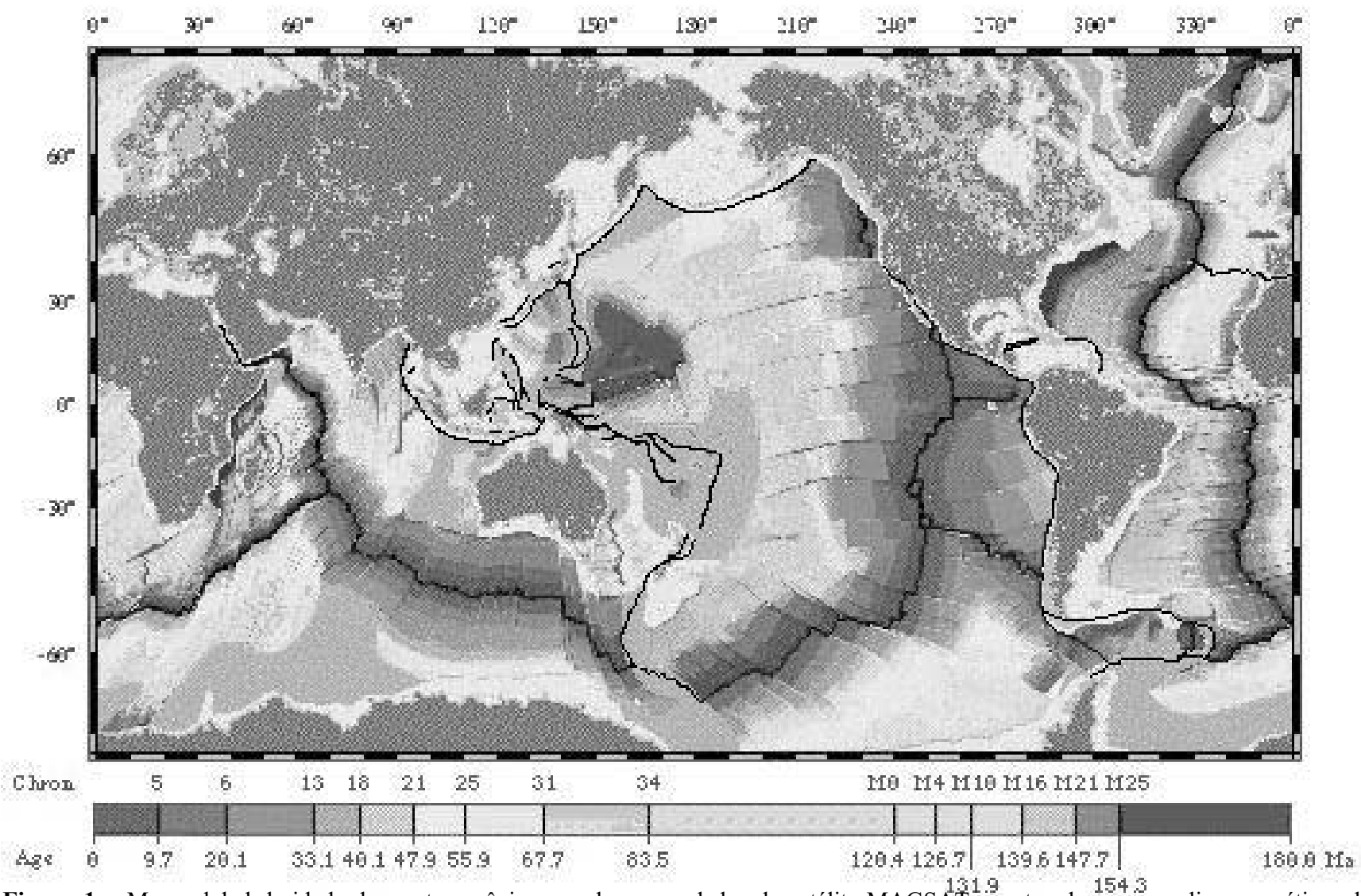

Figura 1 - Mapa global de idade da crosta oceânica com base nos dados do satélite MAGSAT, mostrando as anomalias magnéticas de expansão do fundo oceânico. Modificado de R.D. Müller, W.R. Roest, J.Y. Royer, L.M. Gahagan e J.G. Sclater (1997). Idade em milhões de anos. 

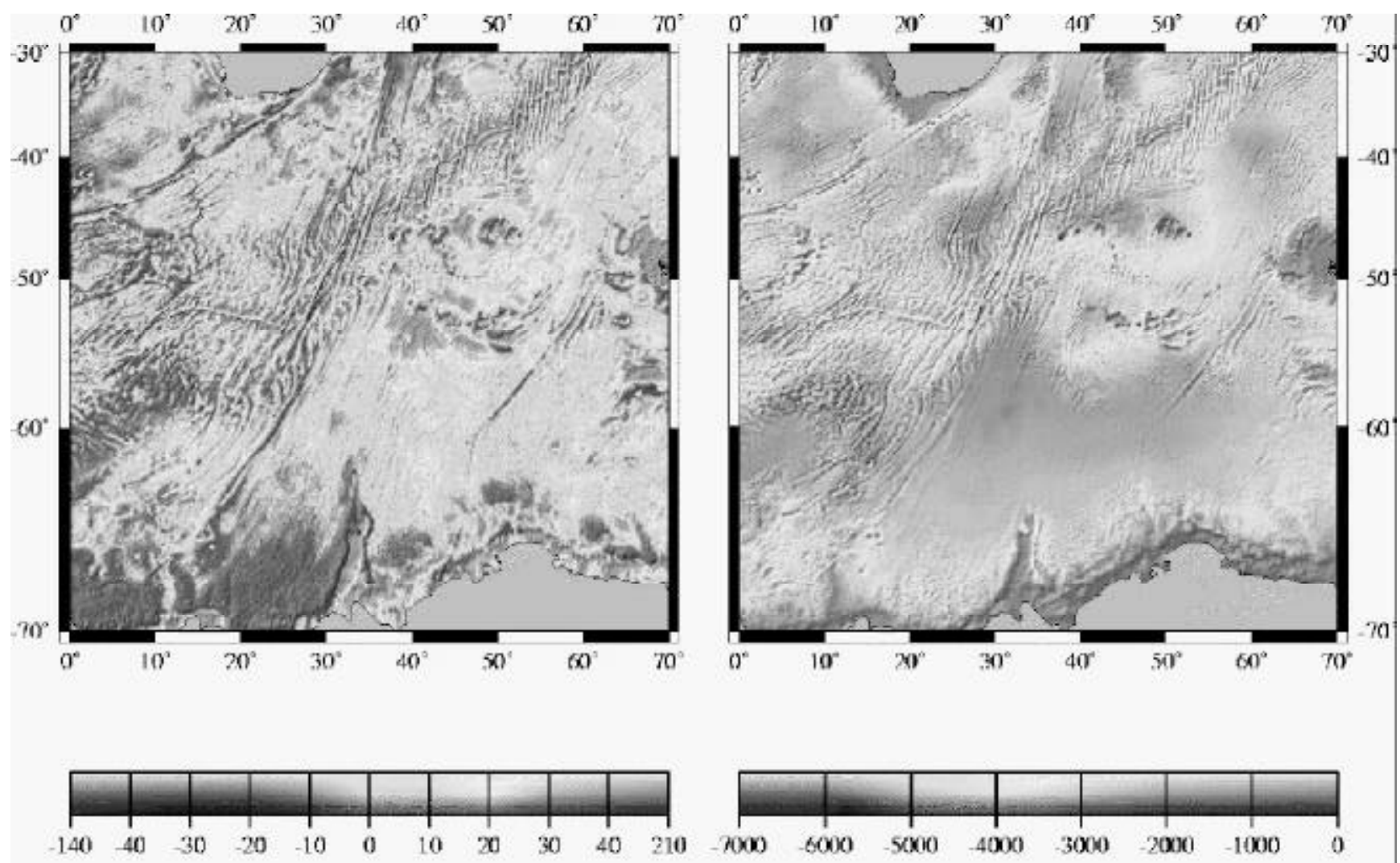

Figura 2 - Mapas regionais da porção limite entre os oceanos Atlântico e Indico derivados da altimetria dos satélites SEASAT, GEOSAT, ERS-1 e TOPEX-POSEIDON. Note que a resolução do dado permite a visualização de feições da ordem de 5-10 km. A direita observase as anomalias gravimétricas Ar-livre e a esquerda observa-se a batimetria predita. Modificado de David T. Sandwell.

Co, Ti, Po e Mn, mostravam-se bem menores que as estimativas. Conseqüentemente, com o aumento dos preços internacionais, tornava-se atraente a exploração dos nódulos polimetálicos, devido ao seu alto grau de pureza. Inicialmente foram mapeadas várias áreas no Oceano Pacífico e umas poucas no Atlântico, principalmente utilizando-se métodos geofísicos acústicos, para caracterização do fundo marinho (batimetria multifeixe, perfilador de sub-fundo de $3.5 \mathrm{kHz}$ e sonografia), além de imagens obtidas em submersíveis. Nesse período, os satélites SEASAT e MAGSAT recobriram todo o planeta, permitindo o mapeamento da magnetoestratigrafia de toda a crosta oceânica (Fig. 1) com uma resolução de aproximadamente $200-300 \mathrm{~km}$.

Somente a partir da década de 90 , com o aumento da precisão proporcionada pelos magnetômetros de césio, passou-se a utilizar a magnetometria como ferramenta para o mapeamento de regiões com alto potencial de recursos minerais marinhos. Estes equipamentos possuem uma precisão da ordem de 0,001 nT, podendo detectar pequenas anomalias magnéticas geradas por rochas com percentual de metais. A magnetometria de alta resolução, por exemplo, medindo as três componentes do campo, vem sendo usada para a caracterização da segmentação da cordilheira e localização dos depósitos de sulfetos maciços. Ainda na década de 90, a integração dos dados obtidos pelos satélites altim étricos SEA SAT, GEOSAT, ERS 1 e TopexPoseidon possibilitou o mapeamento do geóide em todos os oceanos e, conseqüentemente, a estimativa da anomalia gravimétrica ar-livre (Sandwell, $1990 \mathrm{e}$ 1992) e da batimetria predita (Yale, et. al., 1997). Com isto a crosta oceânica vem sendo investigada em detalhe e feições com anomalias gravimétricas de comprimentos de onda maiores que de $20 \mathrm{~km}$ (Fig. 2) podem ser pesquisadas com vistas à definição de províncias com potencial para a exploração de recursos minerais nos oceanos.

Atualmente, com o reaquecimento da indústria de exploração de recursos minerais e com o aumento da precisão dos gravímetros $(0,01 \mathrm{mGal})$ e magnetômetros $(0,001 \mathrm{nT})$; os métodos potenciais 
tornaram-se uma ferramenta útil e econômica para a modelagem de pequenas anomalias gravimétricas e magnetométricas (Fig. 3), em geral, associadas a recursos minerais marinhos (Pierce et al., 1998).

Este trabalho apresenta uma breve discussão sobre os métodos potenciais e sua importância na caracterização dos depósitos minerais marinhos. Inicialmente, apresenta-se a fundamentação teórica como forma de entendimento do método e, em seguida, são citadas algumas aplicações dos métodos

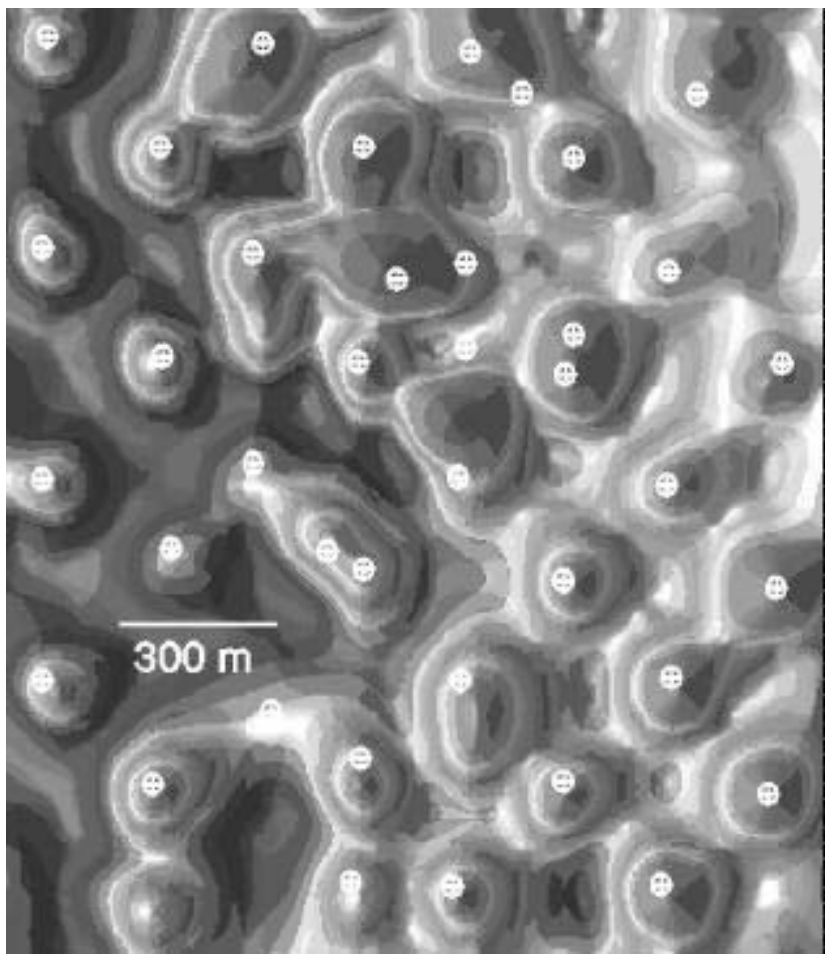

Figura 3 - Mapa de anomalias magnéticas obtido com base em dados de aeromagnetometria de alta resolução. As anomalias magnéticas detectadas têm origem em antigos poços de perfuração (círculos brancos) que foram ababandonados e necessitavam ser remapeados.

potenciais, enfocando principalmente a exploração de hidrocarbonetos em margens continentais.

\section{FUNDAMENTAÇÃO TEÓRICA}

Os métodos potenciais vêm se desenvolvendo fortemente devido à sua ampla aplicabilidade e alta relação custo/benefício, para fins de exploração de recursos minerais marinhos. Tais métodos baseiamse na utilização do campo potencial gravimétrico e/ou magnético da Terra, como fonte de sinal natural que é medido pelos equipamentos geofísicos.
A exploração dos recursos minerais marinhos, através do uso dos métodos potenciais (gravimetria e magnetometria), baseia-se fundamentalmente no processamento e redução destes dados para a interpretação das anomalias gravimétricas e/ou magnetométricas residuais associadas a estes recursos. No entanto, estas anomalias são extremamente pequenas quando comparadas aos campos totais observados, sendo cerca de três à cinco ordens de grandeza menores que o campo geomagnético e gravitacional, respectivamente.

Por este motivo, a obtenção das anomalias residuais requer a quantificação físico-matemática desses campos para que, posteriormente, sua interpretação seja feita por modelagem direta ou inversa. Desta forma, tanto na redução quanto na interpretação das anomalias é fundamental o conhecimento da teoria do potencial que dá suporte a toda a quantificação física necessária.

A teoria do potencial tem origem na Teoria da Gravitação de Newton, sobre os campos gravitacionais, e nos estudos de Gilbert (1540-1603) sobre os campos geomagnéticos, sendo que suas primeiras aplicações para fins de mineração datam de 1556 (Agrícola, 1965).

Tomando como base o campo gravimétrico tem-se, através da Lei da Gravitação Universal, que a força de atração ( F ) entre dois corpos depende da razão direta das massas $\left(m_{1}\right.$ e $\left.m_{2}\right)$ e varia inversamente com o quadrado da distância $(r)$ :

$$
\mathbf{F}=-G \frac{m_{1} m_{2}}{r^{2}} \mathbf{r}_{1},
$$

onde: $G=6,67 \times 10^{-8} d y n-\mathrm{cm}^{2} / \mathrm{g}^{2}$ é a constante de gravitação universal.

Já a força de atração magnética $\left(\mathbf{F}_{\mathbf{m}}\right)$ pode ser obtida a partir da Lei de Coulomb e é muito semelhante à expressão acima, sendo que a constante gravitacional é substituída, de maneira inversa, pela permeabilidade magnética $(\mu)$ do meio físico entre os pólos magnéticos:

$\mathbf{F}_{\mathbf{m}}=\left(\frac{m_{1} m_{2}}{\mu r^{2}}\right) \mathbf{r}_{1}$ 
Portanto, para fins do escopo do artigo, mostraremos de maneira sucinta o tratamento matemático para o caso do campo gravimétrico, ressaltando que o mesmo pode ser estendido para o campo magnético, já que ambos dependem diretamente das massas envolvidas e inversamente do quadrado de suas distâncias, conforme as equações (1) e (2).

Desta forma, considerando $m_{2}$ e $r$ como a massa $(M)$ e o raio $(R)$ da Terra, a aceleração da gravidade $(\mathbf{g})$ é dada por:

$\mathbf{g}=\frac{\mathbf{F}}{m_{2}}=-G \frac{M}{R^{2}} \mathbf{r}_{1}$

Sabe-se então que a aceleração média da gravidade na Terra é de $9,8 \mathrm{~m} / \mathrm{s}^{2}\left(1 \mathrm{~cm} / \mathrm{s}^{2}\right.$ é igual a 1 gal, em homenagem à Galileu), sendo que os gravímetros modernos medem pequenas variações desta aceleração com precisão de $10^{-5} \mathrm{mGal}$.

Um conceito fundamental é que o campo potencial é conservativo, ou seja: o trabalho realizado pela força gravitacional no deslocamento de um corpo entre dois pontos, independe da trajetória do corpo, sendo função apenas de sua posição de partida e chegada. Com isto, se uma massa se move e retorna a sua posição inicial, o trabalho realizado pela força gravitacional é zero, independente da trajetória feita pela massa. Assim, a força gravitacional é um vetor cuja direção se dá ao longo da linha que une as duas massas envolvidas. Este de campo de forças é denominado conservativo e pode ser expresso pelo gradiente de uma função potencial $(U)$ escalar:

$\nabla U(\mathbf{r})=\frac{\mathbf{F}(\mathbf{r})}{m_{2}}=\mathbf{g}(\mathbf{r})$

Basicamente a Teoria do Potencial trata da solução de equações diferenciais utilizando a equação de Laplace e as funções analíticas complexas de Riemann (Kellog, 1953). De acordo com o teorema da divergência de Gauss (Telford et al., 1984), a integral do divergente de um campo vetorial em uma região do espaço é equivalente a integral da componente normal do campo (apontando para fora) sobre a superfície que circunda a região:

$\int_{V} \nabla \cdot \mathbf{g} d v=\int_{S} g_{n} d S$

Se não existe atração de massas (fontes ou sumidouros) dentro do volume as integrais são zero e tem-se então:

$\nabla \cdot \mathbf{g}=0$

sendo que:

$$
\nabla \cdot \mathbf{g}=\nabla \cdot \nabla U=\nabla^{2} U=0,
$$

onde é o potencial escalar satisfazendo a Equação de Laplace em um espaço livre.

A equação de Laplace, para fins de aplicação em Geociências, pode ser representada nos sistemas de coordenadas cartesiano, cilíndrico ou esférico, dependendo da geometria das fontes, obtendo-se respectivamente:

$$
\begin{array}{cc}
\nabla^{2} U=\partial^{2} U / \partial x+\partial^{2} U / \partial y+\partial^{2} U / \partial z=0 & \text { (Cartesiano) } \\
=\frac{1 \partial}{r \partial r}\left(\frac{r \partial U}{\partial r}\right)+\frac{\partial^{2} U}{r^{2} \partial \phi^{2}}+\frac{\partial^{2} U}{\partial z^{2}}=0 & \text { (Cilindrico); } \\
=\frac{\partial}{r^{2} \partial r}\left(r^{2} \frac{\partial U}{\partial r}\right)+\frac{\partial}{r^{2} \sin \theta \partial \theta}\left(\sin \theta \frac{\partial U}{\partial \theta}\right)+ & \\
+\frac{1}{r^{2} \sin ^{2} \theta}\left(\frac{\partial^{2} U}{\partial \phi^{2}}\right)=0 & \text { (Esférico). }
\end{array}
$$

Uma boa referência para a derivação e aplicação dos conceitos da teoria do potencial em geofísica encontra-se em Blakely (1995). Deve-se ressaltar que, a solução da equação de Laplace é a base para o desenvolvimento de modelos físicomatemáticos utilizados na interpretação quantitativa, ou modelagem, das anomalias observadas. Tal modelagem permite a estimativa das densidades, das permeabilidades magnéticas e da geometria das fontes (rochas, estruturas, alvos) que irão definir litologias e estruturas relacionadas aos recursos minerais. Além disto, essa equação é utilizada para descrever processos físicos intimamente relacionados à gênese de recursos minerais sendo os principais deles: i) o fluxo de calor em um meio homogêneo, ii) o equilíbrio de sólidos elásticos e iii) a propagação de correntes 
elétricas em um meio (Carlson \& Jagger, 1959; Turcotte \& Schubert, 1992; Middleton \& Wilcook, 1994 e Reynolds, 1997).

\section{APLICAÇÕES}

As aplicações dos métodos potenciais na prospecção de recursos minerais data da descoberta de hidrocarbonetos na década de 20, no Texas-EUA, através de gravimetria - utilizando a Balança de Torsão de Eötvos - (Gibson \& Millegan, 1998). Desde então, várias aplicações do uso dos métodos potenciais (gravimetria e magnetometria) foram realizadas para fins de exploração de recursos minerais marinhos. Tais aplicações relacionam-se à pesquisas globais, regionais e locais onde pode-se ressaltar os estudos de Patherson \& Reeves (1985), Reynolds et. al. (1987), Keller (1988), Hoog (1989), Chapin (1996), Max et al. (1999) e Burley et al. (2000).

Dentre as aplicações globais e regionais destes métodos, grande parte tem suporte nos dados obtidos por navios oceanográficos e pelo sensoriamento remoto dos satélites MAGSAT, SEASAT, GEOSAT, ERS-1 e TOPEX-POSEIDON (Yale, et. al., 1995). Tais satélites possibilitaram a interpretação regional de anomalias residuais magnéticas ( $\mathrm{Ku} \&$ Sharp, 1983) e gravimétricas (Sandwell, 1990), que são utilizadas para uma série de objetivos de prospecção, como por exemplo: i) para a definição do "potencial e riscos de exploração" de bacias e sub-bacias sedimentares, ii) para definição dos limites entre crosta oceânica e crosta continental e iii) para o mapeamento de novas fronteiras exploratórias. Através da modelagem dessas anomalias pode-se ainda investigar a idade térmica da crosta oceânica e seus processos magmáticos, a espessura dos sedimentos, a morfologia do embasamento, a espessura da crosta e o fluxo de calor superficial. Deve-se destacar que todos estes parâmetros são fundamentais para a definição dos ambientes tectono-magmáticos e sedimentares com potencial para exploração de recursos minerais marinhos.

Atualmente, as aplicações dos métodos potenciais (Leaman, 1994) para a prospecção de recursos minerais marinhos, principalmente petróleo e gás (Shaposhnikov \& Samoletov, 1986), não está limitada apenas ao mapeamento de grandes estruturas geológicas (Cordell \& McCafferty, 1989; Reynolds, et. al., 1990) (Fig. 4). O grande desenvolvimento da aeromagnetometria (Cowan \& Cowan, 1993), da aerogravimetria e da geofísica marinha de alta resolução, com a utilização de gradiômetros magnéticos e gravimétricos (Jekelli, 1988), tem permitido a definição detalhada da geometria de pequenas estruturas (Fig. 5) (Reid et. al., 1990; Macleod et. al., 1993). Os métodos potenciais podem fornecer, portanto informações fundamentais para a exploração e produção de hidrocarbonetos e devem ser integrados a outros métodos geofísicos para sua melhor confiabilidade (Reford, 1980; Lafehr, 1980).

Neste aspecto, uma informação fundamental, por exemplo, é a definição da estrutura de densidades e porosidades de um poço para controle sismoestratigráfico. Atualmente, através de microgravimetria, é possível definir com alta precisão tais parâmetros que são essenciais na conversão tempoprofundidade dos refletores sísmicos utilizados na sísmica de reflexão 3D e 4D. Deve-se ressaltar ainda que, independente do grande desenvolvimento tecnológico dos métodos acústicos, os métodos potenciais têm sido utilizados em escala de detalhe (Machel \& Burton, 1991; Goldhaber \& Reynolds, 1991), no âmbito da geofísica de exploração, inclusive no monitoramento da produção de campos de petróleo e gás.

A partir da década de 90 , os métodos potenciais vêm assumindo um papel de destaque no mapeamento e na exploração de recursos minerais nos oceanos. Suas principais limitações relacionadas às ambigüidades, intrínsecas advindas da teoria do potencial, que geram diferentes soluções nas modelagens, foram aprimoradas através do desenvolvimento de metodologias de interpretação, especialmente aquelas fundamentadas na modelagem por inversão, que incorporam como vínculos outras informações geofísicas e geológicas independentes. Além disto, a ótima relação custo-benefício e a grande disponibilidade de dados nos oceanos (navios e satélites), possibilita seu uso em praticamente todas as províncias marinhas em escala global, regional e até mesmo local. Este fato é particularmente importante, pois possibilita o seu uso integrado, a baixo 


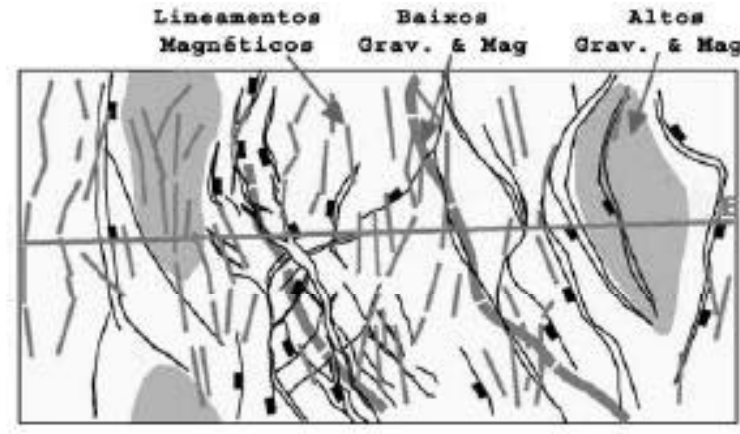

(a)

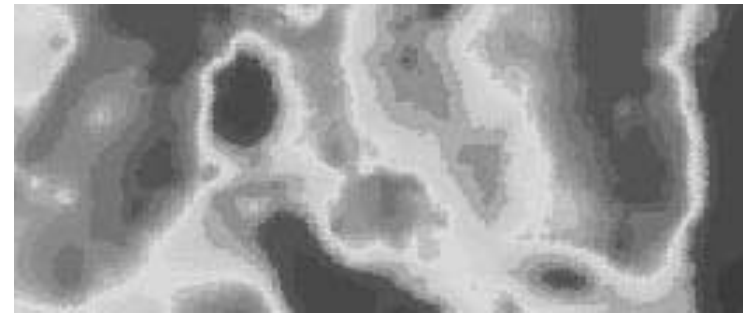

(b)

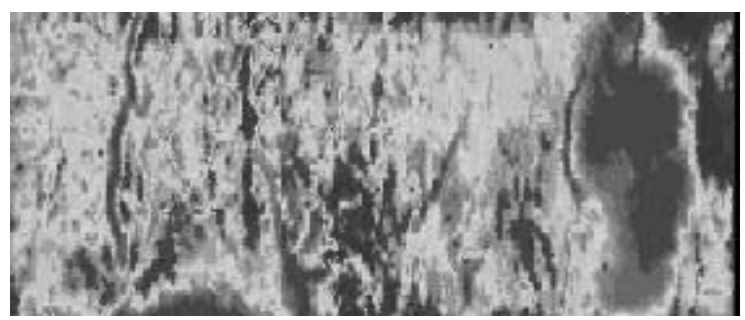

(c)

Figura 4 (A) - Mapa estrutural obtido a partir da interpretação clássica de anomalias magnéticas e gravimétricas em uma província metalífera. Note os blocos do embasamento, os limites das sub-bacias e os lineamentos identificados. (B) Mapa de anomalias gravimétricas residuais (filtro passa-alta) obtidas a partir de dados de aerogravimetria. Note os altos e baixos gravimétricos (porçoes escuras e claras, respectivamente) associados às estruturas geológicas. (C) Mapa de anomalias magnéticas residuais (filtro passa-alta) obtidas a partir de dados de aeromagnetometria. Note os lineamentos estruturais (porções escuras).

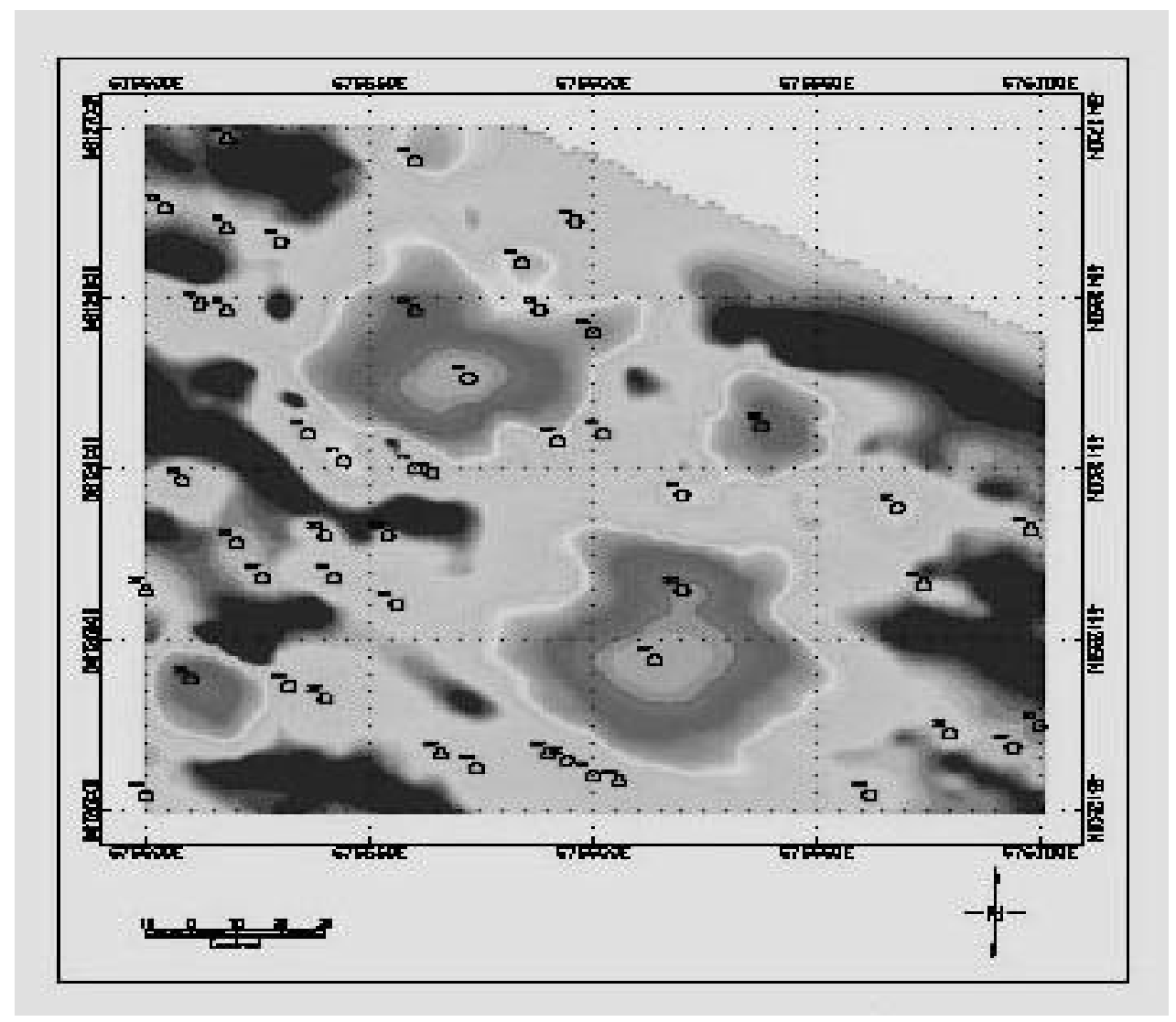

Figura 5 (A) - Mapa de sinal analítico das anomalias magnéticas obtidas com aeromagnetometria de alta resolução em província metalífera. Os pontos marcados representam áreas em prospecção. 

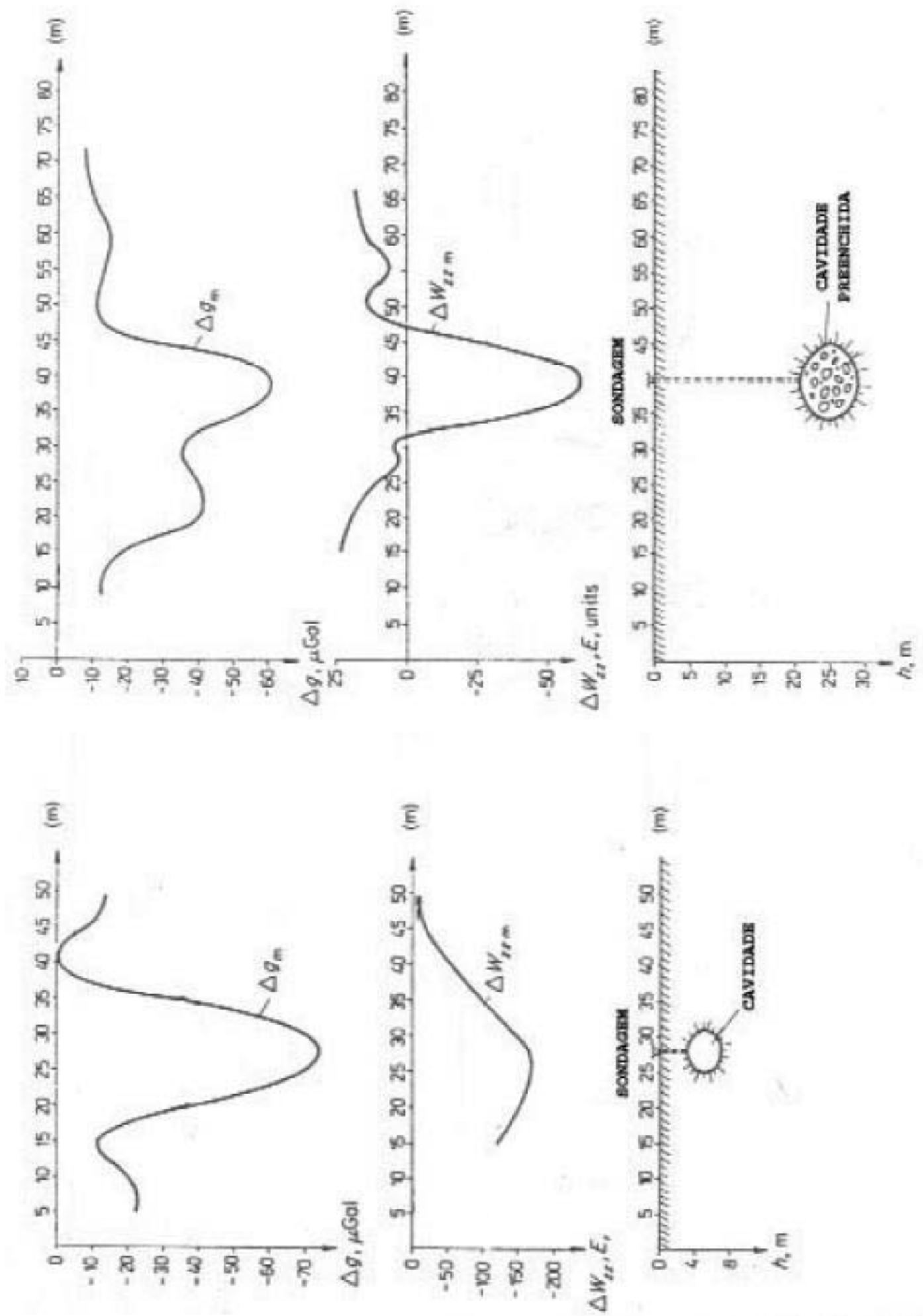

Figura 5 (B) - Anomalias micro-gravimétricas $\left(\nabla g_{m}\right)$ e anomalias de gradiente gravimétrico $\left(\nabla w_{z z m}\right)$ sobre duas pequenas cavidades em rochas carbonáticas. A esquerda a cavidade encontra-se preenchida com ar e a direita preenchida com detritos. Modificado de Fajklewicz (1986). 
custo, na exploração de um amplo espectro de recursos minerais marinhos.

Recentemente, o uso de dados aerogeofísicos de alta resolução nas margens continentais tem propiciado um grande avanço no entendimento da relação entre a geologia continental e a marinha em bacias sedimentares marginais. Como exemplo podese citar um estudo desenvolvido pela FUGRO-LASA, na Bacia de Santos, utilizando dados de aeromagnetometria de alta resolução (Fig. 6). Podese observar (Fig. 6a e Fig. 6b) o alto nível de detalhe proporcionado por estes dados no mapeamento estrutural da bacia, possibilitando a delimitação precisa de sua linha de charneira - identificada pelos limites entre as faixas de alta freqüência espacial ao norte e a porção ao sul dominada por anomalias magnéticas

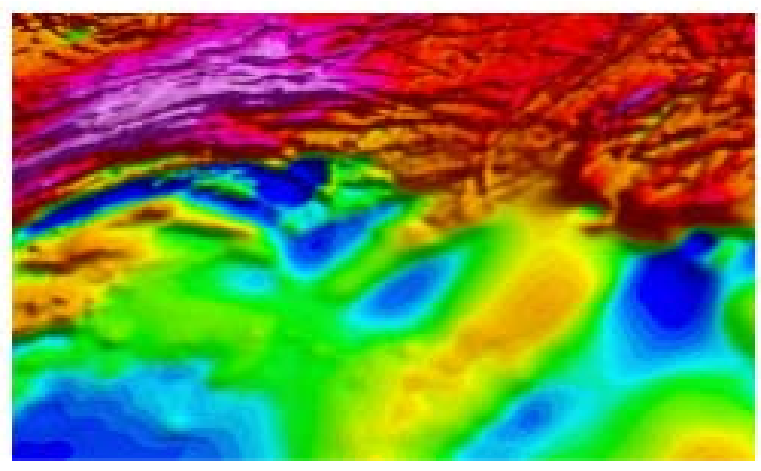

(a)

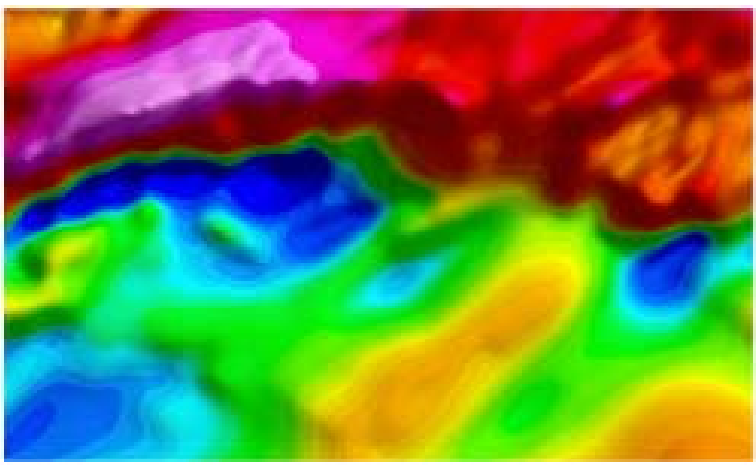

(c) suaves - no mapeamento das sub-bacias, bem identificadas na porção sul do mapa da fase do sinal analítico por sua geometria típica de graben, e no mapeamento da continuidade estrutural da bacia com a Faixa de Dobramentos Ribeira no continente, bem marcada na porção norte do mapa da fase do sinal analítico pela presença de um feixe de lineamentos curvados para o sul.

Na plataforma continental da Namíbia, por exemplo, levantamentos aerogeofísicos de alta resolução como esse são determinantes no mapeamento de paleocanais de drenagem associados aos aluviões que concentram minerais pesados raros e diamantes.

Portanto, as várias aplicações dos métodos potenciais na exploração de recursos minerais

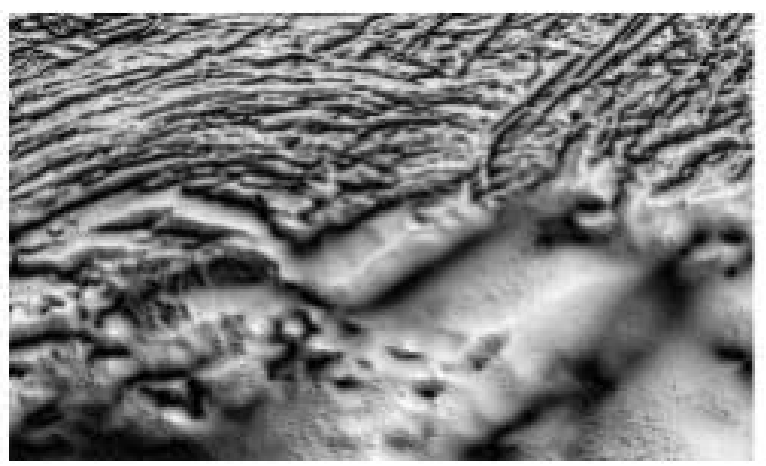

(b)

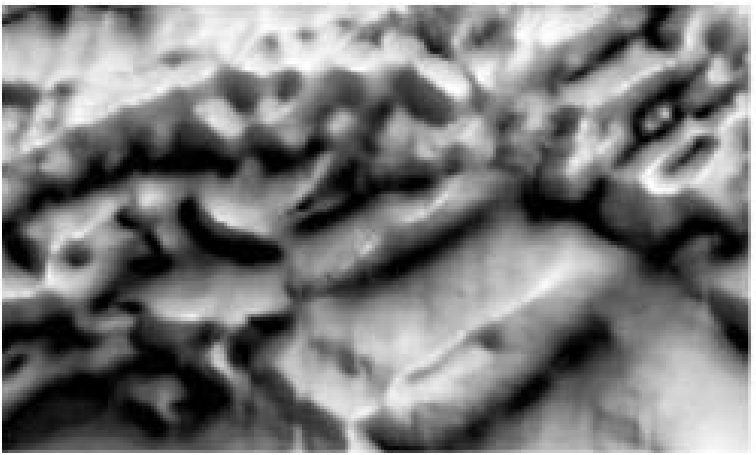

(d)

Figura 6 - Mapas Aeromagnetométricos de uma sub-área da Bacia de Santos, com extensão E-W de aproximadamente 100 km, localizada na plataforma continental ao sul do Rio de Janeiro, Brasil. Estes mapas ilustram a evolução da tecnologia aerogeofísica aplicada à exploração. Topo: levantamento de alta resolução executado em 2001, com espaçamento de $500 \mathrm{~m}$ entre as linhas de vôo e magnetômetros com precisão de 0,001 nT. Base: levantamento regional executado em 1969, com espaçamento de 6 km entre linhas de vôo e magnetômetros com precisão de $1 \mathrm{nT}$. (6a) e (6c) - Mapa de intensidade do campo magnético total; (6b) e (6d) - Fase do sinal analítico do campo magnético total. Cortesia de FUGRO-LASA AIRBORNE SURVEYS. 
marinhos-principalmente em nível de detalhe e alta resolução - trazem novos horizontes para a indústria extrativista, fornecendo informações essenciais para a parametrização dos demais métodos geofísicos, principalmente na locação, exploração e ainda no monitoramento ambiental das jazidas minerais.

\section{REFERÊNCIAS}

AGRICOLA, G., 1965. De re metallica, Dover Publications Inc., New York.

ALLEN, P.A. \& ALLEN, F.D., 1990. Basin Analisys, Principles and Applications, Blackwell Sci. Publ., Oxford London, 451 p.

BLAKELY, R.J., 1995. Potential theory in gravity and magnetic applications, Cambridge Univ. Press.

BURLEY, S.D.; CLARKE, S.; DODDS,A.; FRIELINGSDORF, J.; HUGGINS, P.; RICHARDS, A.; WARBURTON, I.C. \& WILLIAMS, G., 2000. New insights on petroleum migration from the application of 4D basin modelling in oil and gas exploration, Journal of Geochemical Exploration, 70, 46570 .

CARSLAW, H.S. \& JAEGER, J.C., 1978. Conduction of Heat in Solids, Oxford University Press, 510 pp.

CHAPIN, D.A., 1996. A deterministic approach toward isostatic gravity residuals: A case study from South America, Geophysics, 61, 10221033.

CORDELL, L. \& McCAFFERTY, A.E., 1989. A terracing operator for physical property mapping with potential field data, Geophysics, 54, 621-634.

COWAN, D.R. \& COWAN, S., 1993. Separation filtering applied to aeromagnetic data, Expl. Geophysics, 24, 429-436.

GIBSON, R.I. \& MILLEGAN, P.S., 1998. Geologic applications of gravity and magnetics: Case histories, SEG Geophysical Ref. Series no 8 and AAPG Studies in Geology no 43, Tulsa, OK, USA, ISBN 1-56080-078-X.

GOLDHABER, M.B. \& REYNOLDS, R.L., 1991. Relations among hydrocarbon reservoirs, epigeneticsulfidization and rock magnetism: Examples from the south Texsa coastal plain, Geophysics, 56, 748-757.

HOOG, R.L.S., 1989. Recent advances in high sensitivityand high resolution aeromagnetics, in Proceedings of Exploration'87, Ontario Geological Survey, Special Volume 3.

JEKELI,C., 1988. The Gravity Gradiometry Survey System (GGSS), EOS, 69, 116-117.

KARNER, G.D. \& DRISCOLL, N.W., 1993. Rift flank topography and extensional basin architecture: formation of Broken Ridge, Southeast Indian Ocean, An. Acad. Bras. Ciênc., 65, Supl.2, 263-94.

KARNER, G.D., \& WATTS, A.B., 1982. "On Isostasy at Atlantic-Type Continental Margins", Jour. Geoph. Res., 87, B4, 292348.

KELLER, G.R., 1988. The development of gravity and magnetic studies, emphasizing articles publishing in the GSA Bulletin, GSA Bull., 100, 469-478.

KELLOG, D., 1953. Foundations of Potential Theory, Dover Publications Inc., New York, $384 \mathrm{pp}$.

KU, C.C. \& SHARP, J.A., 1983. Werner deconvolution for automated magnetic interpretation and its refinement using Marquadt's inverse modelling, Geophysics, 48, 754-774.

LAFEHR, T.R., 1980. Gravity Method, Geophysics, 45, 1634.

LEAMAN, D.E., 1994. Criteria for evaluation of potential field interpretations, First Break, 12, 181-191.

MACHEL, H.G. \& BURTON, E.A., 1991. Causes and spatial distribution of anomalous magnetization in hydrocarbon seepage environments, AAPG Bull, 75, 1864-1876.

MACLEOD, I.N.; JONES, K. \& DAI, T.F., 1993. 3-D Analytic signal in the interpretation of total magnetic field data at low magnetic latitudes, Exploration Geophysics, 24, 679-688.

MAX, M.D.; GHIDELLA, M.; KOVACS, L.; PATERLINI, M. \& VALLADARES, J.A., 1999. Geology of the Argentine Continental Shelf and Margin from Aeromagnetic Survey, Marine and Petroleum Geology, 16, 41-64. 
McKENZIE, D., 1978. Some Remarks on the Developments of Sedimentary Basins, Eart Planet. Sci. Lett., 40, 25-32.

MIDDLETON, G. V.\& WILCOCK, P.R., 1994. Mechanics in the Earth and Environmental Sciences, Cambridge University Press, 459 $\mathrm{pp}$

NETTLETON, L.L., 1976. Gravity and magnetics in oil prospecting, McGraw-Hill Book Co.

PATHERSON, N.L. \& REEVES, C.V., 1985. Applications of gravity and magnetic surveys: The state-of-the-art in 1985, Geophysics, 50, 2558

PIERCE, J.W.; ABERCROMBIE, H.J.; CHARTERS, R.A.; De PAOLI, G.R. \& GOOSSER, S.A., 1998. Intrasedimentary magnetization by vertical fluid flow and exotic geochemistry, The Leading Edge, 17, 89-92.

REFORD, M.S., 1980. Magnetic Method, Geophysics, 45, 1640.

REID, A.B.; ALLSOP, J.M., GRANSER, H.; MILLET, A.J. \& SOMERTON, I.W., 1990. Magnetic interpretation in three dimensions using Euler Deconvolution, Geophysics, 55, 80-91.

REYNOLDS, J.M., 1997. An Introduction to Applied and Environmental Geophysics, John Wiley \& Sons Inc., 796 pp.

REYNOLDS, R.L., FISHMAN, N.S., WANTY, R.B. \& GOLDHABER, M.B., 1987. Iron sulfide minerals at Cement oil field, Oklahoma - implications for the magnetic detection of oil fields, Geol. Soc. Am. Bull., 102, 368-380.

REYNOLDS, R.L.; WEBRING, M.; GRAUCH, V.J.S. \& TURTLE, M., 1990. Magnetic forward models of Cement oil field, Oklahoma, based on rock magnetic, geochemical and petrologic constraints, Geophysics, 55, 343-353.
SANDWELL, D., 1990. Geophysical Applications of Satellite Altimetry, Reviews of Geophysics Supplement, p. 132-137.

SANDWELL, D.,1992. Antartic marine gravity field from high density satellite altimetry, Geophys. J. Int., 109, 437-448.

SHAPOSHNIKOV, K.K. \& SAMOLETOV, M.V., 1986. Use of combined gravimetric and aeromagnetic data in exploration for oil and gas pools in West Siberia, Petroleum Geology, 23, 362-368.

SPERLE, M., 1997. Significado Tectônico da Topografia do Sudeste do Brasil e suas Prováveis Relações com a Margem Continental Adjacente, Tese de Doutorado, ON-CNPq/LDEO-Columbia University, Publicação Especial do Observatório Nacional, 169 pp.

TELFORD, W.M.; GELDART, L.P.; SHERIFF, R.E. \& KEYS, D.A., 1984. Applied Geophysics, Cambridge University Press, 860 pp., ISBN 0-521-29146-1.

TURCOTTE, D.L. \& SCHUBERT, G., 1982. Geodynamics: Applications of Continuum Physics to Geological Problems, John Wiley \& Sons Inc., $450 \mathrm{pp}$.

WEISSEL, J.K \& KARNER, G.D., 1989. Flexural uplift of rift flanks due to mechanical unloading of the lithosphere during extension, Jour. Geophys. Res., 94, 13919-50.

YALE, M.M. \& SANDWELL, D., 1997. Global seafloor topography from satellite altimetry and ship depth soundings, Science, 277, 19561963.

YALE, M.M.; SANDWELL, D. \& SMITH, W.H.F., 1995. Comparison of along-track resolution of stacked Geosat, ERS-1 and Topex satellite altimeters, J. Geophys. Res., $100,15117-15127$.

\section{NOTE ABOUT THE AUTHOR}

\section{Marcelo Sperle Dias}

Oceanógrafo (UERJ/1988), Mestre em Geofísica (ON$\mathrm{CNPq} / 1992$ ) e Doutor em Geofísica (ON e Columbia University/1997). Atua nas áreas de oceanografia geológica e geofísica marinha, sendo professor adjunto do Instituto de Geociências da UERJ. É responsável por projetos financiados pelo CNPq e FAPERJ, que englobam pesquisas em exploração de recursos minerais 
e meio ambiente. É pesquisador da FAPERJ e consultor adhoc da FINEP, CNPq, FAPERJ e FACEPE.

Luiz Fernando Santana Braga é graduado em Física (UFRJ/1977), Doutor em Geofísica (OSU-USA/1991).
Atuou como geofísico na Petrobrás por 15 anos. Atuou por mais de cinco anos na indústria aerogeofísica. Atualmente é pesquisador do $\mathrm{ON} /$ MCT e consultor do Grupo Fulgro Airborne Surveys.

\section{Sociedade Brasileira de Geofísica - SBGf}

A SBGf foi fundada em 30 de outubro de 1978, quando contava com 225 sócios, com o objetivo de congregar os geofísicos brasileiros. Hoje a SBGf conta com 1200 sócios ativos, 66 dos quais são estrangeiros.

A $\boldsymbol{S B G f}$ mantém a sua Revista Brasileira de Geofísica cujo objetivo é a publicação de trabalhos científicos e técnicos nesta ciência, particularmente nas áreas de Geofísica da Terra Sólida, Geofísica da Atmosfera, Geofísica Nuclear e Geofísica Aplicada, porém aceita trabalhos de Geodésia, Meteorologia, Oceanografia Física e outras áreas correlatas. Atualmente a Revista Brasileira de Geofísica encontra-se em seu décimo oitavo volume publicado.

Os Congressos da $\boldsymbol{S B G} \boldsymbol{f}$ foram iniciados em 1989. A partir de 1991 estes congressos passaram a denominar-se Internacionais, devido ao grande número de inscrições de trabalhos vindos de outras partes do mundo. A freqüência de realizações destes eventos é de dois em dois anos. Paralelamente aos congressos, é organizada uma feira de amostras de produtos e serviços relacionados à Geofisica, a EXPOGEF.

A SBGf dispõe de uma sede própria, situada na cidade do Rio de Janeiro, onde mantém toda a administração da Sociedade, contando com facilidades tais como, microcomputadores Pentium 200Mhz e Pentium II 450, com impressoras a laser e a cores, máquina de reprografia, telefone e fax. A manutenção desta sede deve-se, não somente às anuidades arrecadadas, mas também graças ao apoio financeiro das empresas de Geofísica associadas.

A SBGf está situada na Avenida Rio Branco 156, sala 2510, no centro da cidade do Rio de Janeiro, CEP 20043-900, TeleFax (021) 2533-0064, e-mail: sbgf@sbgf.org.br 